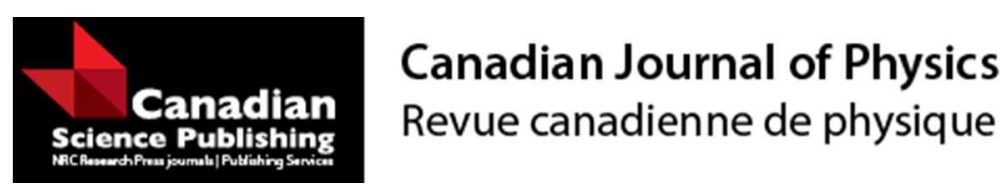

\title{
A microscopic derivation of nuclear collective rotation- vibration model, axially symmetric case
}

\begin{tabular}{|r|l|}
\hline Journal: & Canadian Journal of Physics \\
\hline Manuscript ID & cjp-2015-0371.R2 \\
\hline Manuscript Type: & Article \\
\hline Date Submitted by the Author: & 24-Aug-2015 \\
\hline Complete List of Authors: & Gulshani, P.; NUTECH Services, 3313 Fenwick Cres \\
\hline Keyword: & $\begin{array}{l}\text { collective rotation, vibration, microscopic formulation, variational method, } \\
\text { canonical transformation, rotational band termination, self consistency and } \\
\text { time reversal invariance }\end{array}$ \\
\hline \multicolumn{2}{|l}{} \\
\hline
\end{tabular}

SCHOLARONE ${ }^{\text {IM }}$

Manuscripts 


\title{
A microscopic derivation of nuclear collective rotation-vibration model, axially symmetric case
}

\author{
P. Gulshani \\ NUTECH Services, 3313 Fenwick Crescent, Mississauga, Ontario, Canada L5L 5N1 \\ Tel.\#: 647-975-8233; matlap@bell.net
}

\begin{abstract}
We derive a microscopic version of the successful phenomenological hydrodynamic model of Bohr-Davydov-Faessler-Greiner for collective rotation-vibration motion of an axially symmetric deformed nucleus. The derivation is not limited to small oscillation amplitudes. The nuclear Schrodinger equation is canonically transformed to collective co-ordinates, and then linearized using a constrained variational method. The associated constraints are imposed on the wavefunction rather than on the particle co-ordinates. The approach yields three self-consistent, time-reversal invariant, cranking-type Schrodinger equations for the rotation-vibration and intrinsic motions, and a self-consistency equation. For harmonic oscillator mean-field potentials, these equations are solved in closed forms and applied to the ground-state rotational bands in some axially-symmetric nuclei. The results are compared with those of other models and related measured data.
\end{abstract}

PACS number: 21.60.Ev, 21.60.Fw, 21.60.Jz

Keywords: multi-particle rotation; multi-particle vibration; intrinsic motion; angular momentum; moments of inertia; canonical transformation; constrained variational method; time-reversal invariance; self-consistency; cranking model; axial symmetry; rotational-band termination or cut-off

\section{Introduction}

The phenomenological hydrodynamic rotation-vibration model of Bohr-Davydov-FaesslerGreiner $(B D F G)$ [1-5] has been remarkably successful in predicting the properties of low-lying collective rotation and vibration states in deformed nuclei. The model uses adjustable parameters for the vibration and rotation masses and the first two excitation energies. Therefore, it is of interest to understand the physical nature of the assumptions, approximations, and collective flow dynamics implied by the model, and thereby have more reliable and robust model predictions as alluded to in [6]. In other words, we would like to know how the model Hamiltonian is related to the nuclear Hamiltonian.

To achieve this objective, previous studies [7-20] (refer to [21] for additional references) canonically transformed the nuclear Hamiltonian to a set of collective Euler angles defining the orientation of the nuclear mass quadrupole moment tensor, a set of collective nuclear-shape coordinates chosen to be the diagonal components of the quadrupole moment, and a set of intrinsic 
co-ordinates ${ }^{1}$. The nucleon co-ordinates along the quadrupole-moment-tensor body-fixed axes were subject to constraints, which partially defined the intrinsic co-ordinates. The resulting transformed Hamiltonian decomposed into a sum of three components: an intrinsic, a rotationvibration, and an intrinsic-rotation-vibration coupled component. However, the transformationrelated constraints imposed on the body-fixed particle co-ordinates, the unknown complicated intrinsic co-ordinates, and the complicated nature of the collective and collective-intrinsic coupled components in the transformed Hamiltonian made it extremely difficult to perform any realistic calculation of the energy spectrum and nuclear properties. As discussed in [21], this difficulty was avoided by diagonalizing the $A$-nucleon kinetic energy plus a phenomenological potential energy in a suitably selected subspace of the harmonic oscillator irreducible representation of the non-compact group $\operatorname{SP}(3, R)$, which is a generalization of the compact group $S U(3)$. This approach has been successful using two to six fitting parameters including a pairing interaction in one case [21]. As discussed in footnote 1, alternative, successful microscopic mean-field and angular-momentum projection methods have also been used.

In Section 2 of this article, we circumvent the difficulties associated with the constraints on the particle co-ordinates and the unknown intrinsic co-ordinates by transforming the nuclear Schrodinger equation rather than the Hamiltonian, and by imposing the constraints on the wavefunction rather than on the particle co-ordinates. We thereby deal with the space-fixed particle co-ordinates at each stage of the transformation. We choose the Euler angles such that the Coriolis coupling between the intrinsic and rotation-vibration motions vanishes. The resulting Schrodinger equation is then transformed to a set of collective shape variables. We apply a constrained variational method to linearize the transformed Schrodinger equation for an axially symmetric nucleus. In Section 3, for harmonic oscillator mean-field potentials, the derived governing equations are solved in closed forms and the solutions are simplified. Physical interpretation of the results are also discussed. In Section 4, we discuss the relationship among our, $B D F G$, the variable-moment-of-inertia, and mean-field and angular-momentum projection models. We also review the prescription for evaluating the predicted and measured moments of inertia. In Section 5, we compare the model predictions with the available experimental data and provide physical interpretation of the results. Section 6 concludes the article.

\section{Derivation of microscopic rotation-vibration-intrinsic Schrodinger equation}

We derive the model starting from the microscopic Bohr-Mottelson unified rotational model derived in [26,27] as follows.

We use the Bohr-Mottelson unified rotational-model product wavefunction:

\footnotetext{
${ }^{1}$ Collective Hamiltonians have also been derived using mean-field approaches, such as time-dependent $H F B$, constrained cranked $H F B$, and generator co-ordinate methods [22,23,24, and references therein] by identifying the collective co-ordinates and Hamiltonian with the expectation values of respectively multipole moment operators and microscopic nuclear Hamiltonian, and subsequently quantizing the collective Hamiltonian using Hamilton's principle and usual quantization rules. Angular momentum projection methods and microscopic interactions have also been used to investigate automatic emergence of nuclear rotational spectrum [22,25].
} 


$$
\Psi_{J M}=\sum_{K=-J}^{J} D_{M K}^{J}\left(\theta_{s}\right) \Phi_{J K}\left(x_{n i}\right)
$$

where: $\boldsymbol{D}_{M K}^{J}$ is the Wigner rotation matrix, $J, M$, and $K$ are respectively the total angular momentum (including spin) quantum number and its $z$-components along respectively the spacefixed axes and quadrupole-moment-tensor-attached axes, whose orientations are given by the three Euler angles $\theta_{s}(s=1,2,3)$, and $x_{n j}(n=1, \ldots, A ; j=1,2,3$, where $A=$ nuclear mass number $)$ is a space-fixed nucleon co-ordinate. We require the non-rotational wavefunction $\Phi_{J K}$ in Eq. (1) to satisfy the constraint:

$$
\hat{J}_{A} \Phi_{K}\left(x_{n i}\right)=0
$$

which states that $\Phi_{J K}$ is a zero angular-momentum eigenstate because it is independent of $\theta_{s}$. However, $\Phi_{J K}$ can depend on the eigenvalues $J$ and $K$. We choose $\theta_{s}$ according to the criterion:

$$
\frac{\partial \theta_{s}}{\partial x_{n j}}=\sum_{k=1}^{3} \chi_{j k}^{s} x_{n k}
$$

(for an arbitrary, non-zero, anti-symmetric matrix $\chi^{s}$ for each $s$ ). The criterion in Eq. (3) together with the constraint in Eq. (2) makes the Coriolis interaction term (coupling the rotation and intrinsic motions in the transformed Schrodinger equation) vanish.

Transforming the nuclear Schrodinger equation to the co-ordinates $\theta_{s}$ and its conjugate angular momentum $\hat{J}_{s}$, we obtain [27]:

$$
\left(-\frac{\mathrm{h}^{2}}{2 M} \sum_{n, j=1}^{A, 3} \frac{\partial^{2}}{\partial x_{n j}^{2}}+\hat{V}-E\right) \Phi_{J K^{\prime}}+\frac{1}{2} \sum_{M, K=-J}^{+J} \sum_{A, B-1}^{3} \Phi_{J K} \mathfrak{J}_{A B}^{r i g^{-1}} \boldsymbol{D}_{M K^{\prime}}^{J^{*}} \hat{J}_{B} \hat{J}_{A} \boldsymbol{D}_{M K}^{J}=0
$$

where $M$ is the nucleon mass, $\hat{V}$ is a rotationally invariant two-body interaction, $\hat{J}_{A}$ is the $A^{\text {th }}$ component of the total angular momentum operator along the $A^{\text {th }}$ co-ordinate axis defined by $\theta_{s}$, and $\mathfrak{J}_{A B}^{r i g^{-1}}$ is the component, along the axes $A$ and $B$, of the inverse of the rigid-flow kinematic moment of inertia tensor defined by:

$$
\mathfrak{I}^{r i g} \equiv \operatorname{Tr}(Q)-Q, \quad \text { and } \quad Q_{j k} \equiv M \sum_{n=1}^{A} x_{n j} x_{n k}
$$

where $Q$ the mass quadrupole-moment tensor. The rigid-flow kinematic moment of inertia appears in Eq. (4) because we have chosen $\theta_{s}$ as in Eq. (3) and hence (when combined with the condition in Eq. (2)) to eliminate the Coriolis interaction term in the transformed Schrodinger equation Eq. (4). As shown in [27], another consequence of the choice of $\theta_{s}$ in Eq. (3) is that the nucleon velocity field has the rigid-flow character: 


$$
V_{n j}^{r o t^{l}}=\left(\stackrel{\mathrm{r}_{\omega}^{l}}{\omega^{l}} \times \stackrel{\mathrm{r}}{x_{n}}\right)_{j}, \quad \omega_{j}^{l} \equiv \mathfrak{J}_{j l}^{r i g^{-1}}
$$

with non-vanishing vorticity, consistently with the rigid-flow moment of inertia in Eq. (4).

The spinless intrinsic state, rigid-flow moment of inertia and velocity field, and zero Coriolis interaction in respectively Eqs. (2), (4), and (6) contrast with the irrotational-flow moment of inertia and two-component (rigid plus irrotational) flow velocity fields in other studies [15,2832], where the intrinsic state carries some angular momentum, and the resulting non-zero Coriolis interaction is determined by a shear (rotating-deforming) operator. The two models would of course yield the same final results if the governing equations could be solved.

Specifically, in our model, the interaction of the intrinsic motion with the oscillations coupled to the centrifugal stretching reduces the rigid-flow kinematic moment of inertia (as shown in Section 5), and in [15, 28-32] studies the shear operator would be expected to increase the irrotational-flow kinematic moment of inertia.

Multiplying Eq. (4) on the left by $\boldsymbol{D}_{M K}^{J}$ and integrating over $\theta_{s}$, we obtain [27] the effective rotation-intrinsic asymmetric rotor Schrodinger equation:

$$
\begin{aligned}
E \Phi_{J K}= & \left\{-\frac{\mathrm{h}^{2}}{2 M} \sum_{n, j} \frac{\partial^{2}}{\partial x_{n j}^{2}}+V+\frac{\mathrm{h}^{2}}{4}\left(\mathfrak{J}_{11}^{r i g^{-1}}+\mathfrak{J}_{22}^{r i g^{-1}}\right)\left[J(J+1)-K^{2}\right]+\frac{\mathrm{h}^{2} K^{2}}{2} \mathfrak{J}_{33}^{r i g^{-1}}\right\} \Phi_{J K} \\
& +\frac{\mathrm{h}^{2}}{8}\left(\mathfrak{J}_{11}^{r i g^{-1}}-\mathfrak{J}_{22}^{r i g^{-1}}-2 i \mathfrak{I}_{12}^{r i g^{-1}}\right) \sqrt{(J+K+2)(J-K-1)(J+K+1)(J-K)} \Phi_{J K+2} \\
& +\frac{\mathrm{h}^{2}}{8}\left(\mathfrak{J}_{11}^{r i g^{-1}}-\mathfrak{J}_{22}^{r i g^{-1}}+2 i \mathfrak{I}_{12}^{r i g^{-1}}\right) \sqrt{(J-K+2)(J+K-1)(J-K+1)(J+K)} \Phi_{J K-2} \\
& +\frac{\mathrm{h}^{2}}{4}(2 K-1)\left(\mathfrak{J}_{13}^{r i g^{-1}}-i \mathfrak{I}_{23}^{r i g^{-1}}\right) \sqrt{(J+K+1)(J-K)} \Phi_{J K+1} \\
& +\frac{\mathrm{h}^{2}}{4}(2 K+1)\left(\mathfrak{J}_{13}^{r i g^{-1}}+i \mathfrak{I}_{23}^{r i g^{-1}}\right) \sqrt{(J-K+1)(J+K)} \Phi_{J K-1}
\end{aligned}
$$

The off-diagonal elements $\mathfrak{J}_{A B}^{r i g^{-1}}$ (or equivalently $\left.Q_{A B}\right)(A \neq B)$ are generally small (in fact their averages generally vanish), and in this article they are ignored. In this article, we are also considering only axially symmetric deformed even-even nuclei so that $\left\langle\mathfrak{J}_{11}^{r i g-1}\right\rangle=\left\langle\mathfrak{J}_{22}^{\text {rig-1 }}\right\rangle$ at all values of $J$ and $K$, where the brackets indicate expectation values. Eq. (7) then reduces to:

$$
\left\{-\frac{\mathrm{h}^{2}}{2 M} \sum_{n, j} \frac{\partial^{2}}{\partial x_{n j}^{2}}+V+\frac{\mathrm{h}^{2}}{2 \mathfrak{J}_{11}^{r i g}}\left[J(J+1)-K^{2}\right]+\frac{\mathrm{h}^{2} K^{2}}{2 \mathfrak{J}_{33}^{r i g}}\right\} \Phi_{J K}=E \Phi_{J K}
$$

We now transform Eq. (8) to the collective vibration (rigid-flow-moment-of inertia) coordinates defined by: 


$$
R_{1} \equiv \frac{\mathfrak{J}_{11}^{\text {rig }}}{M}=\sum_{n=1}^{A}\left(y_{n}^{\prime 2}+z_{n}^{\prime 2}\right), \quad R_{2} \equiv \frac{\mathfrak{J}_{22}^{\text {rig }}}{M}=\sum_{n=1}^{A}\left(x_{n}^{\prime 2}+z_{n}^{\prime 2}\right), \quad R_{3} \equiv \frac{\mathfrak{J}_{33}^{\text {rig }}}{M}=\sum_{n=1}^{A}\left(x_{n}^{\prime 2}+y_{n}^{\prime 2}\right)
$$

(where the prime superscript indicates the co-ordinates along the body-fixed axes ${ }^{2}$ given by the transformation $x_{n j}=\sum_{A=1}^{3} R_{A j}\left(\theta_{s}\right) x_{n A}^{\prime}$ where $R_{A j}$ are the elements of an orthogonal matrix). In transforming Eq. (8) to the co-ordinates $R_{1}, R_{2}$, and $R_{3}$ in Eq. (9), we use the product wavefunction:

$$
\Phi_{J K}=F_{1}\left(R_{1}\right) \cdot F_{2}\left(R_{2}\right) \cdot F_{3}\left(R_{3}\right) \cdot \phi_{\mathrm{JK}}\left(x_{n k}\right)
$$

where the spherically symmetric ${ }^{3}$ intrinsic (such as shell-model or $H F B$ ) wavefunction $\phi_{\mathrm{JK}}$ is subject to the constraints:

$$
\frac{\partial}{\partial R_{1}} \phi_{\mathrm{JK}}=0, \quad \frac{\partial}{\partial R_{2}} \phi_{\mathrm{JK}}=0, \quad \frac{\partial}{\partial R_{3}} \phi_{\mathrm{JK}}=0
$$

and we obtain the result [27]:

$$
\begin{aligned}
\sum_{n=1}^{A} \frac{\partial^{2}}{\partial x_{n 1}^{2}} \Phi_{J K}= & 2 A\left(F_{1} F_{3} \frac{d F_{2}}{d R_{2}}+F_{1} F_{2} \frac{d F_{3}}{d R_{3}}\right) \cdot \phi_{J K} \\
& +\sum_{n=1}^{A} x_{n 1}^{2}\left(4 F_{1} F_{3} \frac{d^{2} F_{2}}{d R_{2}^{2}}+8 F_{1} \frac{d F_{2}}{d R_{2}} \cdot \frac{d F_{3}}{d R_{3}}+4 F_{1} F_{2} \frac{d^{2} F_{3}}{d R_{3}^{2}}\right) \cdot \phi_{J K} \\
& +4\left(F_{1} F_{3} \frac{d F_{2}}{d R_{2}}+F_{1} F_{2} \frac{d F_{3}}{d R_{3}}\right) \cdot \sum_{n=1}^{A} x_{n 1} \frac{\partial}{x_{n 1}} \phi_{J K}+F_{1} F_{2} F_{3} \cdot \sum_{n=1}^{A} \frac{\partial^{2}}{\partial x_{n 1}^{2}} \phi_{J K}
\end{aligned}
$$

and similar expressions for the derivatives of $\Phi_{J K}$ with respect to $x_{n 2}$ and $x_{n 3}$. To derive Eq. (12) we have neglected the off-diagonal elements of $Q_{A B}(A \neq B)$ and chosen the arbitrary non-zero anti-symmetric matrix $\chi^{s}$ in Eq. (3) to satisfy the condition:

$$
\chi_{A B}^{s} \omega_{B A}^{s} C_{B A}^{\prime}=-\chi_{A C}^{s} \omega_{C A}^{s} C_{C A}^{\prime}
$$

where $A, B$, and $C=1,2,3$ are in cyclic order, and

$$
\omega_{A B}^{s} \equiv \sum_{j=1}^{3} \frac{\partial R_{A j}}{\partial \theta_{s}} R_{B j}, \quad C_{B C}^{\prime} \equiv \sum_{n=1}^{A}\left(x_{n B}^{\prime 2}-x_{n C}^{\prime 2}\right)
$$

\footnotetext{
${ }^{2} R_{1}, R_{2}, R_{3}$ can be and are chosen independent collective co-ordinates because $A-3$ of the particle co-ordinates $x_{n A}^{\prime}$ are independent of each other.

${ }^{3}$ The prescriptions in Eqs. (10) and (11) extract the quadrupole-moment distribution part of the wavefunction $\Phi_{J K}$ leaving the intrinsic wavefunction $\phi_{\mathrm{JK}}$ spherically symmetric, noting that higher order deformations are found empirically to be relatively small $[1,32]$ and hence are usually not considered at not too high angular momenta.
} 
For $\hat{V}$ in Eq. (8) we use the harmonic-oscillator mean-field potential:

$$
\hat{V}=\frac{M \omega_{\mathrm{int}}^{2}}{2} \sum_{n=1}^{A} r_{n}^{2}+\frac{M \omega_{v 1}^{2}}{2} R_{1}+\frac{M \omega_{v 1}^{2}}{2} R_{2}+\frac{M \omega_{v 3}^{2}}{2} R_{3}
$$

where $r_{n}^{2} \equiv x_{n}^{2}+y_{n}^{2}+z_{n}^{2}$, the first term is the restoring potential for the intrinsic system and remaining terms are the restoring potentials for oscillations in the collective rigid-flow moment of inertia variables $R_{1}, R_{2}$, and $R_{3}$, and where, for application to an axially-symmetric deformed nucleus, we have chosen the oscillation frequencies $\omega_{v 1}$ and $\omega_{v 2}$ to be the same ${ }^{4}$.

Substituting Eqs. (12) and (14) into Eq. (8), we obtain a transformed Schrodinger equation where the collective oscillations in each of the three spatial directions are functionally coupled to each other and to the intrinsic motion. To reduce this coupling to an algebraic coupling effectively linearizing the equation, we apply to the transformed Schrodinger equation a constrained variational method as done previously [34,35]. To achieve this goal, we take the expectation value of the equation. In the resulting equation, we then do the following: (i) we ignore, in the present model, nuclear incompressibility, (ii) we impose the following axialsymmetry conditions on the collective-oscillation wavefunctions (consistently with axially symmetric potential in Eq. (14)): $\left\langle F_{1}\left|R_{1}\right| F_{1}\right\rangle=\left\langle F_{2}\left|R_{2}\right| F_{2}\right\rangle,\left\langle F_{1}\left|\partial / \partial R_{1}\right| F_{1}\right\rangle=\left\langle F_{2}\left|\partial / \partial R_{2}\right| F_{2}\right\rangle$, $\left\langle F_{1}\left|R_{1} \partial^{2} / \partial^{2} R_{1}\right| F_{1}\right\rangle=\left\langle F_{2}\left|R_{2} \partial^{2} / \partial^{2} R_{2}\right| F_{2}\right\rangle$, i.e., the oscillations in the spatial directions 1 and 2 are assumed to be identical and in phase, at all values of $J$ and $K^{5}$, (iii) we ignore any coupling between the collective oscillations in any two spatial directions, and (iv) we vary separately each of the wavefunctions $F_{1}^{*}, F_{2}^{*}, F_{3}^{*}$, and $\phi_{\mathrm{JK}}^{*}$ (i.e., we use the Rayleigh-Ritz variational method) subject to the normalization and energy minimization conditions: $\left\langle F_{1} \mid F_{1}\right\rangle=\left\langle F_{2} \mid F_{2}\right\rangle=\left\langle F_{3} \mid F_{3}\right\rangle=\left\langle\phi_{\mathrm{JK}} \mid \phi_{\mathrm{JK}}\right\rangle=1, \partial E / \partial F_{1}^{*}=\partial E / \partial F_{2}^{*}=\partial E / \partial F_{3}^{*}=\partial E / \partial \phi_{\mathrm{JK}}^{*}=0$, for arbitrary variations in $F_{1}^{*}, F_{2}^{*}, F_{3}^{*}$, and $\phi_{\mathrm{JK}}^{*}$. We then obtain the following three self-consistent, timereversal invariant cranking-type Schrodinger equations and a self-consistency equation:

\footnotetext{
${ }^{4}$ To satisfy the constraint in Eqs. (11), we also need to add a two-body (such as separable monopole-monopole) interactions to the right-hand-side of Eq. (14) as we did in [34,35]. In this article, we do not do so for simplicity and to gain physical insight into the results and because, using the method in [34,35], it can be shown that the effects of the constraints in Eqs. (11) are relatively small. We also satisfy partially the constraint in Eq. (2) by using a spherically-symmetric intrinsic state $\phi_{\mathrm{JK}}$ and placing each pair of nucleons of opposite spins in a pair of lowest time-reversed single-particle orbitals; also refer to footnote 2.

${ }^{5}$ Axial symmetry is assumed because, at moderate values of the angular momentum, most nuclei are found to be axially symmetric, and hence this case is commonly analyzed. Axial symmetry also simplifies the analysis and facilitates gaining physical insight. This assumption does not preclude a transition from a prolate to an oblate shape and vice versa. The model governing equations can just as easily be solved without assuming axial symmetry.
} 


$$
\begin{aligned}
& \left(R_{1}^{2} \frac{d^{2}}{d R_{1}^{2}}+\frac{a_{+}}{2} R_{1} \frac{d}{d R_{1}}-\frac{J(J+1)-K^{2}}{8}+\varepsilon_{1} R_{1}-\frac{b_{v 1}^{2}}{4} R_{1}^{2}\right)\left|F_{1}\right\rangle=0 \\
& {\left[R_{3}^{2} \frac{d^{2}}{d R_{3}^{2}}+a_{-} R_{3} \frac{d}{d R_{3}}-\frac{K^{2}}{4}+\varepsilon_{3} R_{3}-\frac{b_{v 3}^{2}}{4} R_{3}^{2}\right]\left|F_{3}\right\rangle=0} \\
& \left(-\sum_{n=1}^{A} \nabla_{n}^{2}+\left(\beta_{1}+\beta_{3}\right) \cdot \ddot{B}^{0}+\left(\beta_{1}-\beta_{3}\right) \cdot \dddot{B}_{3}^{0}+b_{i n t}^{2} \sum_{n=1}^{A} r_{n}^{2}-\varepsilon_{\text {int }}\right)\left|\phi_{J K}\right\rangle=0 \\
& a_{+} \cdot \beta_{1}+a_{-} \cdot \beta_{3}=\varepsilon_{s}
\end{aligned}
$$

where:

$$
\begin{gathered}
b_{v 1} \equiv \frac{M \omega_{v 1}}{\mathrm{~h}}, \quad b_{v 3} \equiv \frac{M \omega_{v 3}}{\mathrm{~h}}, \quad b_{i n t} \equiv \frac{M \omega_{i n t}}{\mathrm{~h}}, \quad a_{1} \equiv\left\langle\phi_{J K} \mid \not \beta \phi \phi_{J K}\right\rangle, \quad a_{3} \equiv\left\langle\phi_{J K}\left|B_{3}^{0}\right| \phi_{J K}\right\rangle, \\
a_{+} \equiv a_{1}+a_{3}, \quad a_{-} \equiv a_{1}-a_{3}, \quad \beta_{1} \equiv-4\left\langle F_{1}\left|\frac{d}{d R_{1}}\right| F_{1}\right\rangle, \quad \beta_{3} \equiv-4\left\langle F_{3}\left|\frac{d}{d R_{3}}\right| F_{3}\right\rangle \\
\not B=\frac{1}{2} \sum_{n, j=1}^{A, 3}\left(x_{n j} \frac{\partial}{\partial x_{n j}}+\frac{\partial}{\partial x_{n j}} x_{n j}\right), \quad B_{3}^{0} \equiv \frac{1}{2} \sum_{n=1}^{A}\left(x_{n 3} \frac{\partial}{\partial x_{n 3}}+\frac{\partial}{\partial x_{n 3}} x_{n 3}\right)
\end{gathered}
$$

and $\varepsilon_{1}, \varepsilon_{3}, \varepsilon_{i n t}$, and $\varepsilon_{s}$ are functions of the reduced energy $\varepsilon \equiv 2 M E / \mathrm{h}^{2}$ and other system parameters.

\section{Solution of Eqs. (15), (16), (17), and (18)}

The dilation-compression operator $B_{3}^{0}$ in Eq. (17) mixes states of different angular momenta. To enable us to use a single intrinsic antisymmetrized single-particle product wavefuntion and, hence simplify the analysis and obtain physical insight, we set, in this article, $B_{3}^{0}=\lambda B^{\prime}$ in Eq. (17) (noting that $\left\langle B_{3}^{0}\right\rangle=a_{3},\left\langle B^{\circ}=a_{1}\right.$, and $\lambda=a_{3} / a_{1}$, where $\lambda<1$ since $B^{\prime}$ includes $\mathscr{B}_{3}^{0}$, refer to

Eq. (20)). An estimate of $\lambda$ is given in Eq. (30) below in this section. We then readily obtain, from the literature as discussed in [34,35], the solutions of Eqs. (15), (16), and (17) in closed forms ${ }^{6}$, and use them to evaluate the various parameters, such as $a_{1}, \beta_{1}$, and $\beta_{3}$ in Eqs. (19). In particular, we obtain:

$$
\varepsilon=2 b_{c} \Sigma+4 b_{v 1}\left(2 n_{1}+a_{+} / 2+2 k_{1}\right)-\frac{2 b_{v 1} a_{+}\left(a_{+} / 2-1\right)}{a_{+} / 2+2 k_{1}-1}+2 b_{v 3}\left(2 n_{3}+a_{-}+2 k_{3}\right)-\frac{2 b_{v 3} a_{-}\left(a_{-}-1\right)}{a_{-}+2 k_{3}-1}
$$

where:

\footnotetext{
${ }^{6}$ The solutions of Eqs. (15) and (16) given in this article differ from those of Faessler-Greiner rotation-vibration model $[3,4]$ in three respects: (i) our solutions are not limited to small amplitude oscillations about a mean deformation, (ii) the kinematic moment of inertia is not an adjustable parameter but rather is a dynamical variable (specifically is the rigid-flow moment), and (iii) the interaction between rotation-vibration and intrinsic motions is included.
} 


$$
\begin{gathered}
b_{c} \equiv \sqrt{b_{i n t}^{2}+\beta_{13}^{2} / 4}, \quad \beta_{13} \equiv(1+\lambda) \beta_{1}+(1-\lambda) \beta_{3}, \quad a_{3}=\lambda a_{1}, \quad a_{+}>2, a_{-}>1 \\
2 k_{1} \equiv-\left(a_{+} / 2-1\right)+\sqrt{\left(a_{+} / 2-1\right)^{2}+J(J+1) / 2}, \\
2 k_{3} \equiv-\left(a_{-}-1\right)+\sqrt{\left(a_{-}-1\right)^{2}+K^{2} / 2}, \quad \sum \equiv \sum_{N=0}^{N_{f}}(N+3 / 2)
\end{gathered}
$$

$n_{1}=0,1,2,3, \ldots \infty$ and $n_{3}=0,1,2,3, \ldots \infty$ are the quantum numbers for the collective oscillations in respectively 1 and 3 spatial directions (and may be considered to describe the so-called beta and gamma band heads), $\Sigma$ is the total oscillator particle-occupation number, $N$ is an oscillator-shell quantum number, and $N_{f}$ is the value of $N$ for the last particle-occupied (Fermi) shell.

The first term on the right-hand side of Eq. (21) for the reduced total energy $\varepsilon$ is the intrinsic-system energy, the second and fourth terms are the energy eigenvalues for the collective oscillations (including the effects of centrifugal stretching $J(J+1)$ and $K^{2}$ in $2 k_{1}$ and $2 k_{3}$ parameters in Eq. (23) in respectively 1 and 3 spatial directions), and the third and fifth terms are the energies arising from the interaction between the collective vibration displacement and intrinsic dilation-compression (i.e., from the terms $-a_{+} \cdot \beta_{1}$ and $-a_{-} \cdot \beta_{3}$ in Eqs. (15) and (16)). The third and fifth terms in Eq. (21) are observed to have always negative values. Therefore, these terms reduce the collective energy and the moment of inertia below the rigid-flow value. The negative energy terms increase in value (i.e., become less negative) with $J$ as the dilationcompression interaction expands the intrinsic system transferring the corresponding energy to the collective motion. This energy transfer increases the collective energy, excitation energy, and the moment of inertia in the states above the first $2^{+}$excited-state. For the ground state, $J=0$ (i.e., for $K=0,2 k_{1}=0,2 k_{3}=0, n_{1}=0$ and $n_{3}=0$ ), and the third and fifth terms in Eq. (21) cancel the second and fourth terms yielding the ground-state energy $\varepsilon_{o}=2 b_{c o} \Sigma$, where $\left.b_{c o} \equiv b_{c}\right|_{J=0}$.

From the solutions of Eqs. (15), (16), (17), and (18) for the ground-state rotational band, for which $K=0,2 k_{3}=0, n_{1}=0$, and $n_{3}=0$, we obtain:

$$
\frac{b_{v 1}\left[a_{1}(1+\lambda) / 2-1\right](1+\lambda)}{a_{1}(1+\lambda) / 2+2 k_{1}-1}+b_{v 1}(1-\lambda) \omega=\frac{a_{1} b_{i n t}}{\sqrt{\Sigma^{2}-a_{1}^{2}}}
$$

where $\omega \equiv b_{v 3} / b_{v 1}$. For given values of $b_{v 1}, b_{i n t}$, and $\omega$ we can solve Eq. (24) together with Eq. (23) numerically. However, we obtain a closed-form solution and more physical insight by assuming that $a_{1}(1+\lambda)>>2$, and ignoring the relatively small term $b_{v 1}(1-\lambda) \omega$ in Eq. (24), whose validity is confirmed by the results of the calculation in Section 5. Eq. (24) then yields the solution: 


$$
a_{1}=\sqrt{\frac{b_{v 1}^{2}(1+\lambda)^{4} \Sigma^{2}-2 b_{i n t}^{2} J(J+1)}{b_{v 1}^{2}(1+\lambda)^{4}+b_{i n t}^{2}(1+\lambda)^{2}}}
$$

Clearly, Eq. (25) has no real solutions at and above the cut-off angular momentum $J_{c}$ given by:

$$
J_{c}\left(J_{c}+1\right)=\frac{b_{v 1}^{2}(1+\lambda)^{4} \Sigma^{2}}{2 b_{i n t}^{2}} \Rightarrow J_{c}=-\frac{1}{2}+\frac{1}{2} \sqrt{1+\frac{2 b_{v 1}^{2}(1+\lambda)^{4} \Sigma^{2}}{b_{i n t}^{2}}}
$$

Therefore, the rotational band is predicted to terminate at and above $J_{c}$ given in Eq. (26). In practice, $J$ is a discrete number and hence, at $J_{c}$, we can ensure that $a_{+}$and $a_{-}$predicted by Eqs. (19), (22), and (25) are sufficiently larger than 2 and 1 respectively as required in Eq. (22).

The results in Eqs. (24), (25), and (26) may be interpreted physically as follows. Eq. (24) arises from the self-consistency that exists between Eqs. (15) and (17) through the parameters $a_{1} \equiv\left\langle\phi_{J K}|B| \phi \phi_{J K}\right\rangle$ and $\beta_{1} \equiv-4\left\langle F_{1}\left|\frac{d}{d R_{1}}\right| F_{1}\right\rangle$ that appear as weights to the collective displacement operator $\frac{d}{d R_{1}}$ and the intrinsic compression operator $B^{\prime}$ in Eqs. (15) and (17) respectively. This self-consistency determines $a_{1}$ in Eq. (25) and the maximum permissible value $J_{c}$ of $J$ in Eq. (26). We can show that Eq. (24) is equivalent to the equation:

$$
\left\langle\phi_{J K}\left|\sum_{n=1}^{A} r_{n}^{2}\right| \phi_{J K}\right\rangle ; \frac{2}{(1+\lambda)^{2}}\left\langle F_{1}\left|R_{1}\right| F_{1}\right\rangle
$$

Eq. (27) requires that the size of the intrinsic system be proportional to that of the collective quadrupole system. Now

$$
\left\langle\phi_{J K}\left|\sum_{n=1}^{A} r_{n}^{2}\right| \phi_{J K}\right\rangle=\Sigma / 2 b_{c}
$$

(so that $\left\langle\phi_{J K}\left|\sum_{n=1}^{A} r_{n}^{2}\right| \phi_{J K}\right\rangle$ is proportional to $\Sigma$ ), and

$$
\left\langle F_{1}\left|R_{1}\right| F_{1}\right\rangle ; \sqrt{\left(a_{+} / 2\right)^{2}+J(J+1) / 2} / b_{v 1}
$$

(so that $\left\langle F_{1}\left|R_{1}\right| F_{1}\right\rangle$ increases with $J$, a centrifugal stretching effect). It follows that, at some critical value $J_{c}$ of $J$, the size of the collective system would exceed that of the intrinsic system, and the self-consistency condition in Eq. (27) is violated. At this point, the rotational motion 
breaks down and the rotational band terminates. Near the band termination, $a_{1}$ in Eq. (25) and $\beta_{1}$ become small and hence the interaction between the collective and intrinsic systems becomes small. However, the moment inertia remains less than the rigid-flow value, as the results in Section 5 shows.

Eq. (26) indicates that $J_{c}$ is essentially proportional to $\Sigma$. Therefore, a change in the intrinsic system configuration (generated by the inclusion of a pairing interaction, more rigorous treatment of the constraint in Eq. (2) and the operator $B_{3}^{0}$ in Eq. (17), inclusion of the currentlyneglected cross terms in the transformed kinetic energy in Eq. (12), etc.) would alter the value of $\Sigma$ and hence $J_{c}$, as speculated in [36].

We can evaluate $\lambda$ by determining $a_{3}$ from its definition in Eq. (19) as we have done for $a_{1}$. This evaluation is straight-forward but somewhat involved in the spherical-coordinate-system representation for the intrinsic-system wavefunction $\phi_{\mathrm{JK}}$. In Cartesian co-ordinate system, we can easily solve Eq. (17) exactly without resorting to the approximation $B_{3}^{0}=\lambda B^{\circ}$, and for $J=0$, we find:

$$
\lambda=\left.\frac{a_{3}}{a_{1}}\right|_{J=0}=\left[1+(1+\omega) \cdot \frac{\Sigma_{1}+\Sigma_{2}}{2 \Sigma_{3}} \sqrt{\frac{b_{i n t}^{2}+4 b_{v 1}^{2}}{b_{i n t}^{2}+b_{v 1}^{2}(1+\omega)^{2}}}\right]^{-1}
$$

where the $\Sigma_{k} \equiv \sum_{n_{k}=0}^{n_{j k}}\left(n_{k}+1 / 2\right),(k=1,2,3)$, is the total oscillator particle-occupation number in the $k^{\text {th }}$ spatial direction, and $n_{f k}$ is the value of the oscillator quantum number $n_{k}$ at the Fermi level. We use Eq. (30) as an approximation for $\lambda$ at all $J$ values.

For the ground-state rotational band (i.e., for $K=0,2 k_{3}=0, n_{1}=0$, and $n_{3}=0$ ), the total energy in Eq. (21) becomes:

$$
\varepsilon=2 b_{c} \Sigma+4 b_{v 1}\left(a_{+} / 2+2 k_{1}\right)-\frac{2 b_{v 1} a_{+}\left(a_{+} / 2-1\right)}{a_{+} / 2+2 k_{1}-1}
$$

where $b_{c}, a_{+}$, and $2 k_{1}$ are given by Eqs. (19), (22), and (25). The excitation energy of a member of the ground-state rotational band is defined by:

$$
\Delta E_{J} \equiv \frac{\mathrm{h}^{2}}{2 M}[\varepsilon(J)-\varepsilon(J=0)]
$$

\section{Definition of moment of inertia and comparison of the collective and other models}

For large values of $a_{1}$ and hence $a_{+}$, we may expand the energies in Eqs. (31) and (32) in powers of $J(J+1)$ to obtain the well-known phenomenological Variable-Moment-of-Inertia model expression [5,37-41]: 


$$
\begin{aligned}
\Delta E_{J} & \equiv \frac{\mathrm{h}^{2}}{2 M}[\varepsilon(J)-\varepsilon(J=0)] \\
& =A J(J+1)-B J^{2}(J+1)^{2}+C J^{3}(J+1)^{3}+\ldots . \\
& \equiv \frac{\mathrm{h}^{2} J(J+1)}{2 \mathfrak{I}_{J}}
\end{aligned}
$$

where the coefficients $A, B, C$, etc. are functions of $b_{v 1}, b_{i n t}, \omega$, and $\Sigma$, and the moment of inertia $\mathfrak{I}_{J}$ is a function of $b_{v 1}, b_{\text {int }}, \omega, \Sigma$, and $J$. An expansion resembling that in Eq. (33) was also obtained in the Faessler-Greiner rotation-vibration model as a result of including the vibration-rotation interaction (a second-order term in their expansion in the deformation parameters) [4].

Generally, the moment of inertia $\mathfrak{I}_{J}$ for a given member of a rotational band with angular momentum $J$ is defined by [41]:

$$
\begin{aligned}
\Delta E_{J}-\Delta E_{J-2} & \equiv \frac{\mathrm{h}^{2}}{2 \mathfrak{I}_{J}}[J(J+1)-(J-2)(J-1)] \\
& =\frac{\mathrm{h}^{2}}{2 \mathfrak{I}_{J}}(4 J-2)
\end{aligned}
$$

Therefore,

$$
\frac{2 \mathfrak{I}_{J}}{\mathrm{~h}^{2}}=\frac{4 J-2}{\Delta E_{J}-\Delta E_{J-2}}(\mathrm{MeV})^{-1}
$$

where $\Delta E_{J}$ is either the predicted or measured excitation energy.

Except for the terms containing the off-diagonal elements $\mathfrak{\Im}_{A B}^{r i g^{-1}}(A \neq B)$ and rigid-flow moment of inertia $\mathfrak{J}_{A A}^{r i g^{-1}}$, the rotational kinetic energy term in Eq. (7) is identical to that in the Faessler-Greiner rotation-vibration model [4].

In our present model, the axial-symmetry conditions imposed, in Section 2, on the variables $R_{1}$ and $R_{2}$, their derivatives, and the products of these (i.e., the oscillations in the spatial directions 1 and 2 being identical and in-phase) are assumed to hold at all values of $J$ and $K$. That is, the shape of the nucleus remains axially symmetric as it rotates and oscillates about this shape. To describe tri-axial deviations from the axially symmetric shape, we must consider the full three tri-axial oscillation equations rather than only the two Eqs. (15) and (16). Our present model ignores nuclear incompressibility. On the other hand, the Faessler-Greiner rotation- 
vibration model [4] describes an incompressible, irrotational-flow ${ }^{7}$ rotation and small-amplitude oscillations of a tri-axial nucleus about an axially-symmetric deformed equilibrium shape. That is, in the Faessler-Greiner model, $\mathfrak{J}_{11}^{r i g-1} \neq \mathfrak{I}_{22}^{r i g^{-1}} \neq \mathfrak{J}_{33}^{r i g^{-1}}$, and hence the nucleus is slightly tri-axial as it rotates and vibrates.

Except for: (a) the extra terms in $a_{+}$and $a_{-}$in Eqs. (15) and (16) representing the coupling of the oscillations to the intrinsic motion and for the rigid-flow inertia variables, and (b) the comments made above on incompressibility, axial symmetry versus tri-axiallity, and the factor of 2 difference between Eqs. (15) and (16) arising from imposing the axial symmetry condition, Eqs. (15) and (16) resemble those in the Bohr-Davydov-Faessler-Greiner rotation-vibration model [1-5] when a transformation to the co-ordinate $R=\rho^{2}$ is made (refer also to footnote 6).

However, only the second and fourth terms in Eq. (21) have some resemblance to the terms in the energy eigenvalue in the Faessler-Greiner model [4]. Even these terms are different since angular momentum appears under the square-root sign (in $2 k_{1}$ in Eq. (23)). The main reason for this radical sign is that we do not limit the analysis to small amplitude oscillations.

The model in this article offers an alternative physical mechanisms for gradual and large increases in the moment of inertia and for a band termination to those provided by other models. Nevertheless, the mechanisms in this and other models closely parallel one another as the following description attempts to show.

In the successful microscopic mean-field approaches (such as time-dependent $H F B$, constrained cranked $H F B$, generator co-ordinate, and projected $H F B$ methods $[22,43,23,24$, and references therein]) for a description nuclear vibration-rotational motion, the Coriolis interaction is a dominant force. These models predict [22,42, and references therein] that the experimentally observed gradual increase with $J$ in the moment of inertia, and hence the resulting deviation of the rotational energy spectrum from $J(J+1)$ rule for a strongly-bound system, is primarily due to a Coriolis-force-induced weakening of nuclear pairing correlation and gradual alignment of the axes of many quasi-particle orbits along the rotation axis $[43,44]$ with increasing $J$ (known as Coriolis anti-pairing or $C A P$ effect). Centrifugal stretching and the resulting increase in deformation play a minor role in this deviation. At sufficiently high $J$, the $C A P$ effect breaks up a two paired nucleons in the highest angular momentum orbitals and align their spins along the rotation axis (known as the rotation alignment or $R A L$ effect), causing a sudden increase in the moment of inertia (known as the back-bending effect). But the moment of inertia remains less than the rigid-flow (or rigid-body) value consistent with the measured moment of inertia.

\footnotetext{
${ }^{7}$ However, the constant factors in the rotation and vibration masses used in the Faessler-Greiner model are not those for irrotational flow but are rather fitted to the measured excitation energies of the first excited $2^{+}$states. The impact of this course of action and its possible inconsistency need to be studied because the measured inertia masses do not have irrotational-flow character. The kinematic moment of inertia in the Faessler-Greiner model is proportional to the square of the deformation parameter, whereas the rigid-flow kinematic moment of inertia in our model is insensitive to very small value of the deformation.
} 
Eventually, $R A L$ causes the disappearance or quenching of the pairing correlation, and hence a transition from a super-fluid to a normal fluid nuclear state, and a corresponding increase in the moment of inertia. However, the moment of inertia still remains less than the rigid-flow value (due to the shell structure and internal counter currents [45], which the cranking model seems to predict [46,47 and references therein]) consistent with the measured moment. For nuclei with moderate deformations in this fully aligned state with the maximum angular momentum, the rotation is not collective but rather has an independent particle nature. In this state, the collective ground-state rotational band therefore terminates, and the nucleus acquires an oblate particle distribution about the rotation axis, which therefore becomes the symmetry axis as well $[42,48,49]$, similarly to a classical (macroscopic) object. In this non-collective state, the collective nuclear deformation therefore disappears and $E 2$ transitions vanish or become small $[50,51,52]$. Excited-state beta and gamma rotational bands (including triaxially shaped states) would have different band-termination $J$ values and would require a separate consideration. For nuclei with large deformations, the nuclear spin remains less than the maximum as the nucleus continues to deform (due to the centrifugal stretching) towards necking and fission with increasing angular momentum [49].

In the model derived in this article, the Coriolis interaction in the nuclear Schrodinger Eqs. (4), (8), (15), (16), and (17), which couples the rotational and non-rotational degrees of freedom, has been transformed away and eliminated completely by the choice of the rotating frame. This elimination requires the constraint in Eq. (2), i.e., that the intrinsic state have zero angular momentum. (This state is obtained by placing each pair of nucleons in a pair of timereversed orbitals, or in other words, by having a state of paired nucleon.) The elimination of the Coriolis interaction results in: (i) the rigid-flow kinematic moment of inertia ("kinematic" is defined as the value of the moment that does not include contributions from rotation-vibrationintrinsic interaction), and (ii) an interaction among the rotation, vibration, and intrinsic motions that is the product of collective vibration displacement and intrinsic (radial) compression/ dilation, given by the second term on the L.H.S. of Eqs. (15) and (16), and the second and third terms on L.H.S. of Eq. (17). As mentioned in Section 3 in relation to the result in Eq. (21), this interaction term generates the negative third and fifth terms in the excitation energy in Eq. (21) and Eq. (28). This interaction, and its consequential energy exchange between the vibrationrotation and intrinsic systems, are responsible for reducing the rigid-flow kinematic moment of inertia to the measured value in the excited rotational band states. (This reduction in the rigidflow moment of inertia, arising from the interaction of the rotation-vibration and paired-nucleon intrinsic motions, parallels that achieved in the self-consistent cranking model by the addition of nuclear pairing correlations.)

The negative energy terms mentioned in the preceding paragraph become smaller with $J$ (as $a_{+}$and $2 k_{1}$ in Eqs. (21) and (28) decreases and increases respectively, reducing the collective and intrinsic radial displacements). Therefore, the moment of inertia in the ground-state rotational band begins to increase with $J$ in the states above the first excited $2^{+}$state. At a sufficiently high $J$, the centrifugal stretching causes the size of the quadrupole collective system 
to exceed that of the intrinsic system, and hence a breakdown of the self-consistency between these two systems (refer to the physical interpretation of Eqs. (24), (25), and (26) in Section 3). At this $\left(J_{c}\right)$ critical value of $J$, the quadrupole deformation and hence $E 2$ transitions vanish or become small, and the rotational band terminates. However, the moment of inertia remains less than the rigid-flow value (as Tables 1 and 2 show). As can be seen in Eq. (25), the value of $J_{c}$ depends on the intrinsic-system particle-occupation number $\Sigma$. Therefore, a change in the intrinsic-system configuration (generated by the inclusion of a pairing interaction, more rigorous treatment of the constraint in Eq. (2) and the operator $B_{3}^{0}$ in Eq. (17), inclusion of the currentlyignored cross terms in the transformed kinetic energy in Eq. (12), etc.) would alter the value of $\Sigma$ and hence of the excitation energy, moment of inertia, and $J_{c}$. Therefore, in some nuclei, $J_{c}$ may become so large that the band practically would not terminate. Furthermore, the constraint in Eq. (2) may be replaced by $\left\langle\phi_{J K}\left|{ }^{\mathrm{r}}\right| \phi_{J K}\right\rangle=0$ in the first approximation. This constraint is similar to the cranking-model type of constraint in the microscopic mean-field models [23]. This constraint would introduce a Coriolis interaction term into Eq. (17) resulting in the excitation of paired particles into higher angular momentum orbitals, and hence in higher values of $\Sigma, a_{1}$, excitation energy, and moment of inertia. This excitation increases the quadrupole size of the system and hence the moment of inertia (refer to Eqs. (27), (28), and (29)). The paired-particle excitation and the resulting aforementioned system changes would depend on $J$ through the action of the term $\left(\beta_{1}+\beta_{3}\right) \cdot \mathscr{B}^{\circ}+\left(\beta_{1}-\beta_{3}\right) \cdot \mathscr{B}_{3}^{0}$ in Eq. (17) because $\beta_{1}$ and $\beta_{3}$ depend on $J$.

Furthermore, when the currently-neglected cross terms in the transformed kinetic energy in Eq. (12) are included in Eqs. (15) and (16), Eqs. (24) and (25) may become more complex possibly giving several values of $a_{1}$ and hence the excitation energy for a given $J$ or angular frequency, similarly to that in the $H F B$ approach [22]. This result could also give rise to backbending.

The above sequence of physical mechanisms and the corresponding changes in the moment of inertia in the collective and mean-field models are summarized and compared in the table below.

\section{$\underline{\text { Self-consistent rotating mean-field models }}$}

- The Coriolis interaction yields the rigidbody moment of inertia in absence of a pairing interaction.

- Inclusion of pairing interaction reduces the rigid-body moment of inertia to the measured value.

\section{$\underline{\text { Collective model }}$}

The elimination of the Coriolis interaction yields the rigid-flow kinematic moment of inertia.

Interaction of vibrationrotation with a paired nucleon intrinsic state reduces the 
- With increasing $J$, Coriolis interaction weakens the pairing correlation and gradually aligns many quasi-particle orbitals along the the rotation axis (the $C A P$ effect).
- At sufficiently high $J$, the $C A P$ effect breaks up a two paired nucleons in the highest angular momentum orbitals and align their spins along the rotation axis (the rotation alignment or $R A L$ effect), causing a sudden increase in the moment of inertia (the back-bending effect). Eventually, $R A L$ causes a transition from a super-fluid to a normal fluid non-collective state with a corresponding increase in the moment of inertia, which remains less than the rigid-flow moment. For nuclei with moderate deformations, the groundstate rotational band terminates in this noncollective fully-aligned state with the maximum angular momentum. In this state, the rotation generates an oblate particle distribution around the rotation axis, which therefore becomes the symmetry axis as well. For nuclei with large deformations, the angular momentum remains less than the maximum as the deformation increases with $J$ towards necking and fission. Description of excited-state rotational bands requires a separate consideration. rigid-flow moment to the measured value.

The interaction between the collective and intrinsic system decreases with $J$ causing the reduction in the moment of inertia to become smaller with $J$. Therefore, the moment of inertia begins to increase towards the rigidflow value in the states above the $J=2^{+}$state.

At a sufficiently high $J$, the centrifugal stretching causes the size of the quadrupole collective system to exceed that of the intrinsic system, and hence the breakdown of the selfconsistency between these two systems. At this $\left(J_{c}\right)$ critical $J$, the band terminates. But the moment of inertia remains less than the rigid-flow value. Since $J_{c}$ depends on $\Sigma$, a change in the intrinsic-system configuration (generated by the inclusion of a pairing interaction, more rigorous treatment of the constraint in Eq. (2) and the operator $B_{3}^{0}$ in Eq. (17), inclusion of the the neglected cross terms in the transformed kinetic energy, etc.) would alter the value of $\Sigma$ and hence of the excitation energy, moment of inertia, and $J_{c}$. Thus, for 
some nuclei, $J_{c}$ may become so large that the band would not terminate. Furthermore, the zero angular-momentum constraint may cause pairedparticle excitation, increasing the moment of inertia. Inclusion of the currentlyneglected kinetic energy cross terms may render $a_{1}$ and hence the energy and moment of energy multivalued for a given $J$.

\section{Application of model to some nuclei}

In this section, we apply the model developed in Section 3 to predict the properties of the ground-state rotational bands in nuclei ${ }_{4}^{8} \mathrm{Be},{ }_{6}^{12} \mathrm{C},{ }_{10}^{20} \mathrm{Ne},{ }_{12}^{24} \mathrm{Mg},{ }_{14}^{28} \mathrm{Si},{ }_{66}^{162} \mathrm{Dy}$, and ${ }_{68}^{168} \mathrm{Er}$. For each of the nuclei, we determine the ground-state deformation parameter and $\Sigma$ in Eq. (25) from its Nilsson's self-consistent deformed-oscillator particle configuration [49]. The nucleus's oblate or prolate shape is determined from the sign of the measured intrinsic quadrupole moment $[53,54,55]$ and, for consistency, compared with that obtained in Hartree-Fock $(H F)$ and grouptheoretic calculations [56-65]. For the ground-state rotational band, we set $K=0,2 k_{3}=0$, $n_{1}=0$, and $n_{3}=0$, as mentioned in connection with Eqs. (24) and (31). (Excited-state bands, for which $K \neq 0,2 k_{3} \neq 0, n_{1} \neq 0$, and $n_{3} \neq 0$ will be considered in a future study.) The strengths $b_{\text {int }}$ and $b_{v 1}$ in Eq. (19) of the collective potentials in Eq. (14) are determined from matching, as closely as possible, the predicted and measured energies of the first excited $2^{+}$state in the ground-state rotational band, while ensuring that the values of $a_{+}$and $a_{-}$predicted by Eqs. (19), (22) and (25) are sufficiently larger than 2 and 1 respectively (as required in Eq. (22)). This match fixes $b_{i n t}$ and $b_{v 1}$ and the first excited $2^{+}$state energy or equivalently (though Eq. (35)) the moment of inertia, but it does not fix the energies and moments of inertia of the higher excited states of the rotational band, noting that the model moment of inertia is a dynamical quantity and not a fixed parameter, unlike that in phenomenological models. It may be possible to determine $b_{i n t}$ and $b_{v 1}$ from other considerations. To calculate the electric quadrupole moment and $B(E 2)$, we need to know the ratio $\omega \equiv b_{v 3} / b_{v 1}$ of the strengths of collective potentials in Eq. (14) and (19). We determine $\omega$ from equating the ground-state (i.e., $J=0$ ) intrinsic quadrupole moment predicted by the model to that evaluated using the Nilsson deformed self-consistent oscillator model [49]. For more accurate results, we are free to use the ground state calculated by any model, such as $H F B$ and modern mean-field approaches, because the model is concerned only with excited states and not the ground state. Alternatively, we can use measured intrinsic 
quadrupole moment or $B(E 2)$ value as we have done for the nuclei ${ }_{66}^{162} \mathrm{Dy}$ and ${ }_{68}^{168} \mathrm{Er}$ because for these nuclei the simple Nilsson's model is not sufficiently accurate.

The results of the analysis show that the approximations used to obtain Eq. (25) from Eq. (24) are justified.

The calculation shows that the dilation interaction (i.e., the $-a_{+} \cdot \beta_{1}$ terms in Eqs. (15) and (17)) between the oscillations and intrinsic-system motion, which yields the negative energy term in the excited-state energy in Eq. (31), is essential for reducing the ground-state energy such that the excitation energy in Eq. (32) is a positive quantity at all $J$. The variation of the interaction term with $J$ is also essential for yielding the observed increment in the excitation energy with $J$.

The results in Tables 1 and 2 show that the model predicts reasonably well the excitation energy $\Delta E_{J}$ (within $20 \%$ for ${ }_{4}^{8} \mathrm{Be}$ and $16 \%$ for ${ }_{10}^{20} \mathrm{Ne}$, and within $5 \%$ or less for the other nuclei in Tables 1 and 2). For ${ }_{66}^{162} D y$ in Table 2, the predicted excitation energy is within $20 \%$ or less of the measured excitation energy. This excitation energy is progressively over-predicted with $J$. As a consequent of its definition in Eq. (35), the moment of inertia is progressively underpredicted. This difference may be due to a partial satisfaction of the zero angular momentum constraint in Eq. (2), the approximate treatment of the operator $B_{3}^{\circ}$ in Eq. (17), and the neglect of the cross terms in the transformed kinetic energy in Eq. (12). This discrepancy will be explored in a future study.

For ${ }_{68}^{168} \mathrm{Er}$ in Table 2, the agreement between the predicted and measured excitation energies is excellent, and is close to that predicted by the Faessler-Greiner rotation-vibration model [70]. Not that the Faessler-Greiner model includes the rotation-vibration interaction arising from triaxiallity, whereas our model excludes this interaction. This difference in the two models may be traceable to the difference in the kinematic moments of inertia in the two models and the presence of oscillation-intrinsic system interaction in our model, as discussed in the footnotes 6 and 7.

The rotational-band cut-off angular momentum $J_{c}$ given in Eq. (26) for all the nuclei in Tables 1 and 2 is predicted at the same value above which no experimentally observed excited states belonging to the ground-state rotational band can be found.

The excitation energy and hence (due to its definition in Eq. (35)) the (dynamic) moment of inertia is generally reasonably well predicted. The reduction in the model moment of inertia from its kinematic rigid-flow value to the measured value is achieved in the model by the interaction between rotation-vibration and intrinsic motions without using any pairing interaction (a similar reduction in the moment of inertia without using a pairing interaction has also been obtained in $S P(3, R)$ model [21]). On the other hand, a number of previous studies, such as that in [71], have used a pairing interaction to reduce the self-consistent-cranking-model-predicted 
rigid-flow moment of inertia to that observed experimentally. However, the approaches in our and the cranking model may not be too different and may be closely related when we observe that in our model the oscillations interact with a paired-nucleon isotropic intrinsic state and in the cranking model the Coriolis interaction acts on a paired deformed nuclear state (also refer to the discussion in Section 4). The measured and predicted moments of inertia increase gradually with $J$. They are a factor of two or less (going from the light to heavier nuclei) smaller than the rigid-flow ${ }^{8}$ moment of inertia. As discussed in Section 3, this result indicates that the model interaction between vibration-displacement and intrinsic dilation expands the intrinsic system. The transfer of the associated energy from the intrinsic system to the collective motion increases the excitation energy and hence reduces the rigid-flow kinematic moment of inertia, and alters the kinematic rigid-flow velocity field.

Tables 1 and 2 show that the measured and predicted moments of inertia for most of the nuclei are four and many-times-more smaller than the rigid-body moment of inertia (going from light to heavier nuclei), as expected.

The predicted ground-state quadrupole moment $e Q_{o}$ is reasonably close to the measured value. For prolate nuclei, $e Q_{o}$ increases with $J$ from its $J=0$ value to higher prolate values as a consequence of centrifugal stretching (i.e., $J(J+1)$ term in Eqs. (15) and (23)). This stretching occurs only in the $R_{1}$ (i.e., $z$ axis) direction (refer to Eq. (9)) because, in the ground-state rotational band, $K=0$ and $2 k_{3}=0$ and hence no centrifugal stretching in the $R_{3}$ (i.e., y axis) direction can occur (as seen from Eqs. (9), (16) and (23)). Therefore, in oblate nuclei ${ }_{6}^{12} C$ and ${ }_{14}^{28} \mathrm{Si}$ in their ground-state rotational band, the quadrupole moment transitions, at some higher $J$ values, to a prolate shape. Table 1 presents the available measured $e Q_{o}$ and electric quadrupole probability transition rate $B(E 2)$ values. In most cases, the predicted $e Q_{o}$ and $B(E 2)$ are somewhat close to the measured values when the measurement uncertainties are considered. However, we expect discrepancies between the predicted and measured variations in $e Q_{o}$ and hence $B(E 2)$ with $J$. In particular, the model predicts that, in all the nuclei considered, the $B(E 2)$ increases monotonically with $J$ in prolate nuclei, whereas the measured $B(E 2)$ in some nuclei seems to increase at low values of $J$ and decrease at higher values of $J$. Possible reasons for this discrepancy may be the: (i) neglect in the governing Eqs. (15) and (16) of the cross terms in Eq. (12) that couple the oscillations in a pair of spatial directions, (ii) partial satisfaction of the zero angular momentum constraint in Eq. (2), (iii) approximate treatment of the operator $B_{3}^{0}$ in Eq. (17), and (iv) neglect of nuclear incompressibility and tri-axiallity and the resulting band mixing, as discussed in Section 4.

\section{Concluding remarks}

In this article, we have derived a microscopic version of the remarkably successful phenomenological hydrodynamic Bohr-Davydov-Faessler-Greiner nuclear rotation-vibration

\footnotetext{
${ }^{8}$ Note that rigid-flow does not required the nucleons to be frozen at various locations as is the case in a rigid body.
} 
model. The microscopic model accounts completely for the effects of the Coriolis interaction, resulting in the kinematic rigid-flow moment of inertia, nucleon velocity field, and vibration mass. The model identifies a dilation-compression operator for the interaction between the rotation-vibration and intrinsic motion, and a rotational-band cut-off angular momentum.

The present model describes a compressible nucleus that remains strictly axially symmetric while it rotates and oscillates about this shape, with no limit on the oscillation amplitude. On the other hand, the Faessler-Greiner rotation-vibration model describes the collective rotation and small-amplitude oscillations of an incompressible irrotational tri-axial nucleus about an axiallysymmetric deformed equilibrium shape.

The model is derived from a canonical transformation of the nuclear Schrodinger equation to collective rotation angles chosen such that the Coriolis interaction term in the transformed Schrodinger equation vanishes, yielding a tri-axial rigid-flow rotor Schrodinger equation. This equation is then transformed to collective vibration co-ordinates chosen to be the three principal components of the rigid-flow moment of inertia tensor. The resulting equation is then linearized using a constrained variational method to obtain, for an axially symmetric nucleus, three coupled self-consistent, time-reversal invariant cranking-type Schrodinger equations for the intrinsic and two rotation-vibration motions, and a self-consistency equation. We are at liberty to choose any (such a shell or $H F B$ model) isotropic intrinsic wavefunction, subject to zero-angular momentum and zero collective-vibration displacement constraints.

The above transformations differ from the those in previous studies in the following ways: (i) the Schrodinger equation rather the Hamiltonian is transformed, (ii) the associated constraints are imposed on the wavefunction rather on the nucleon co-ordinates, (iii) one deals with the space-fixed particle coordinates and avoids intractable intrinsic co-ordinates and constraints, and (iv) the Coriolis interaction term in the transformed Schrodinger equation is eliminated by a judicious choice of the rotation angles.

For harmonic oscillator-type mean-field potentials for the vibration and intrinsic systems, and for an axially symmetric nucleus, we solve the model cranking-type Schrodiner equations and self-consistency equation in closed forms. For ground-state rotational band, the solutions are simplified to facilitate physical understanding, and in particular to determine an analytic expression for rotational-band cut-off angular momentum. (The simplifications are justified by the results of the calculations.) The simplified equations, with the intrinsic and collective oscillator potential energy strengths as two adjustable parameters, are applied to the ground-state rotational band of ${ }_{4}^{8} \mathrm{Be},{ }_{6}^{12} \mathrm{C},{ }_{10}^{20} \mathrm{Ne},{ }_{12}^{24} \mathrm{Mg},{ }_{14}^{28} \mathrm{Si},{ }_{66}^{162} \mathrm{Dy}$, and ${ }_{68}^{168} \mathrm{Er}$. The results are encouraging.

The excitation energies are reasonably well predicted. For ${ }_{68}^{168} \mathrm{Er}$ and ${ }_{66}^{162} \mathrm{Dy}$ nuclei, the agreement between the predicted and measured excitation energies is excellent (although for ${ }_{66}^{162} D y$, the excitation energy is progressively over-predicted with $J$ up to a maximum of $20 \%$ ). The cut-off angular momentum is well predicted for all the nuclei studied. This cut-off occurs 
when self-consistency between the collective and intrinsic systems breaks down due to centrifugal stretching.

The measured and predicted dynamic moments of inertia agree reasonably closely without using any pairing interaction in the zero-spin intrinsic system. The measured and predicted moments of inertia are a factor of two or less smaller than the rigid-flow moment of inertia and much smaller than the rigid-body moment of inertia. The calculation results show that dilationcompression interaction between the rotation-oscillation and intrinsic motions increases the size of the intrinsic system transferring the corresponding energy to the collective motion. This increases the excitation energy and hence reduces the rigid-flow kinematic moment of inertia and alters the rigid-flow nucleon velocity field. However, the contribution of this interaction decreases with $J$, and hence the moment of inertia begins to increase in states above the first excited $2^{+}$state in the ground-state rotational band. Therefore, the model offers a mechanism for reducing the rigid-flow moment of inertia without using a pairing interaction. In contrast, pairing has been used in cranking-model calculations to achieve this reduction. There is however, a close parallel between the model mechanisms for the changes in the moment of inertia and those in mean-field models as discussed in Section 4. The dilation-compression mechanism for reducing the kinematic rigid-flow moment of inertia in our model also differs from the shearing mechanism in other canonical-transformation-related models that is expected to increase the kinematic irrotational-flow moment of inertia.

The predicted quadrupole moments and $B(E 2)$ 's agree reasonably well, within the measurement uncertainties, with the available measured data. However, for a more accurate variations of the predicted $e Q_{o}$ and $B(E 2)$ with $J$, we need to examine the effects of: (i) the neglected cross terms in Eq. (12) that couple the oscillations in a pair of spatial directions, (ii) a more rigorous satisfaction of the zero angular momentum constraint in Eq. (2), (iii) a more rigorous treatment of the operator $B_{3}^{0}$ in Eq. (17), and (iv) nuclear incompressibility and triaxiallity.

In future endeavours, we intend to study the impact of the model features (i) to (iv) listed above, and investigate further the nature of the mechanisms for the changes in the moment of inertia. We also intend to apply the model to the ground- and excited-state rotational bands, and examine the phenomena at high angular momentum in axial nuclei in the light, rare-earth, and actinide regions. We plan to generalize the model to the tri-axial case, and study the above phenomena.

\section{Acknowledgement}

The author would like to thank the reviewers for their thorough reading of the manuscript and their valuable comments, particularly on the relationship among physical mechanisms involved in our model and the cranking model. 
Table 1. Predicted/measured excitation energy $\left(\Delta E_{J}\right)$, Cut-off $\boldsymbol{J}\left(J_{c}\right)$, moments of inertia $\left(\mathfrak{I}_{J}\right), e Q_{o}, \boldsymbol{B}(\boldsymbol{E} 2)$

\begin{tabular}{|c|c|c|c|c|c|c|c|}
\hline & $\boldsymbol{J}$ & $\begin{array}{c}\Delta \boldsymbol{E}_{J} \\
(\mathrm{MeV}) \\
\text { model/exp }\end{array}$ & $\begin{array}{c}2 \mathfrak{I}_{J} / \mathrm{h}^{2} \\
(\mathrm{MeV})^{-1} \\
\mathbf{m o d e l} / \mathbf{e x p}\end{array}$ & $\begin{array}{c}2 \mathfrak{I}_{\text {rigflow }} / \mathrm{h}^{2} \\
(\mathrm{MeV})^{-1}\end{array}$ & $\begin{array}{c}2 \mathfrak{I}_{\text {rigbody }} / \mathrm{h}^{2} \\
(\mathrm{MeV})^{-1}\end{array}$ & $\begin{array}{c}\text { Predicted } \\
\boldsymbol{e} \boldsymbol{Q}_{\boldsymbol{o}} / \boldsymbol{B}(\boldsymbol{E 2}) \\
e \mathrm{fm}^{2} / \mathrm{e}^{2} \mathrm{fm}^{4}\end{array}$ & $\begin{array}{c}\text { Measured } \\
\boldsymbol{e} \boldsymbol{Q}_{o} / \boldsymbol{B}(\boldsymbol{E} 2) \\
e f m^{2} / e^{2} f m^{4}\end{array}$ \\
\hline${ }_{4}^{8} B e$ & $\begin{array}{c}2^{+} \\
4^{+} \\
J_{c}=4^{+} \\
\end{array}$ & $\begin{array}{r}3.6 / 2.9 \\
10.5 / 11.4^{*}\end{array}$ & $\begin{array}{l}1.7 / 2.0 \\
2.0 / 1.7\end{array}$ & 3.6 & $\begin{array}{l}5.3 \\
5.7\end{array}$ & $\begin{array}{c}25 / 62 \\
40 / 81 \\
(19 /- \text { at } J=0) \\
\end{array}$ & $\begin{array}{c}19 / 34 \pm 10.5 \\
\text { Qo is for } J=0 \\
\left(H F-B C S \text { pre-diction for } 2^{+}[66]\right)\end{array}$ \\
\hline${ }_{6}^{12} \mathrm{C}$ & $\begin{array}{c}2^{+} \\
4^{+} \\
J_{c}=4^{+}\end{array}$ & $\begin{array}{r}4.7 / 4.4 \\
14.0 / 14.1^{*}\end{array}$ & $\begin{array}{l}1.3 / 1.4 \\
1.5 / 1.5\end{array}$ & $\begin{array}{l}2.5 \\
2.7\end{array}$ & $\begin{array}{l}6.0 \\
6.4\end{array}$ & $\begin{array}{c}-11 / 12 \\
7 / 3 \\
(-17.7 /- \text { at } J=0)\end{array}$ & $\begin{array}{c}-21 \pm 10.5 / 11-99 \text { Qo is for } J=0 \\
{[54]} \\
/ 8.5 \text { for } 2^{+}[56,57]\end{array}$ \\
\hline${ }_{10}^{20} \mathrm{Ne}$ & $\begin{array}{c}2^{+} \\
4^{+} \\
6^{+} \\
8^{+} \\
J_{c}=8^{+}\end{array}$ & $\begin{array}{c}1.4 / 1.6 \\
4.4 / 4.3 \\
8.8 / 8.8 \\
13.8 / 12.0^{*}\end{array}$ & $\begin{array}{l}4.4 / 3.7 \\
4.6 / 5.4 \\
5.0 / 4.9 \\
5.0 / 9.5\end{array}$ & $\begin{array}{l}7.1 \\
7.3 \\
7.6 \\
8.2\end{array}$ & $\begin{array}{l}28.6 \\
29.2 \\
30.3 \\
32.8\end{array}$ & $\begin{array}{c}54 / 290 \\
\\
67 / 233 \\
92 / 86 \\
\\
153 / 997 \\
(49 /- \text { at } J=0)\end{array}$ & $\begin{array}{c}70 \pm\left. 17.5\right|_{\mathrm{J}=0} / 274-762[54], 57 \pm 8 \\
{[57], 480 \pm 8[67]} \\
/ 71 \pm 7[57] \\
/ 66 \pm 8[57] \\
/ 24 \pm 8[57]\end{array}$ \\
\hline${ }_{12}^{24} \mathrm{Mg}$ & $\begin{array}{c}2^{+} \\
4^{+} \\
6^{+} \\
8^{+} \\
J_{c}=8^{+}\end{array}$ & $\begin{array}{r}1.3 / 1.4 \\
4.3 / 4.1 \\
8.5 / 8.1 \\
13.6 / 13.2 *\end{array}$ & $\begin{array}{l}4.6 / 4.4 \\
4.8 / 5.1 \\
5.2 / 5.5 \\
6.0 / 5.9\end{array}$ & $\begin{array}{l}7.4 \\
7.7 \\
8.2\end{array}$ & $\begin{array}{l}35.0 \\
35.7 \\
36.9 \\
39.4\end{array}$ & $\begin{array}{c}30 / 91 \\
46 / 105 \\
74 / 245 \\
134 / 765 \\
(24 /- \text { at } J=0)\end{array}$ & $\begin{array}{c}84 \pm\left. 21\right|_{\mathrm{J}=0} / 395-1097[54] \\
119.3 \pm 25[57], 425 \pm 29[67] \\
/ 95,+21,-16[57] \\
/ 140,+193,-49[57] \\
/ 74,+148,-29[57]\end{array}$ \\
\hline${ }_{14}^{28} \mathrm{Si}$ & $\begin{array}{c}4^{+} \\
6^{+} \\
J_{c}=6^{+}\end{array}$ & $\begin{array}{l}4.1 / 4.6 \\
8.8 / 8.5^{*}\end{array}$ & $\begin{array}{l}4.4 / 4.9 \\
5.4 / 5.6\end{array}$ & $\begin{array}{l}7.6 \\
8.3\end{array}$ & $\begin{array}{l}42.5 \\
46.6\end{array}$ & $\begin{array}{c}-53 / 277 \\
-7 / 3 \\
103 / 482 \\
(-70 /- \text { at } J=0)\end{array}$ & $\begin{array}{c}-38.5 \pm\left. 21\right|_{\mathrm{J}=0} / 30.5-352.2[54] \\
72 \pm 9[57], 317 \pm 17[67] \\
/ 96 \pm 8[57] \\
/ 106 \pm 55[57]\end{array}$ \\
\hline
\end{tabular}

* No measured ground-state rotational-band energy level above this energy is reported in the Table of Isotopes and Nuclear Data Sheets. 
Table 2. Predicted/measured excitation energy $\left(\Delta E_{J}\right)$, Cut-off $\boldsymbol{J}\left(J_{c}\right)$, moments of inertia $\left(\mathfrak{I}_{J}\right)$, e $Q_{o}, \boldsymbol{B}(\boldsymbol{E} 2)$

\begin{tabular}{|c|c|c|c|c|c|c|c|}
\hline & $J$ & $\begin{array}{c}\Delta \boldsymbol{E}_{J} \\
(M e V) \\
\text { model/exp }\end{array}$ & $\begin{array}{c}2 \mathfrak{I}_{J} / \mathrm{h}^{2} \\
(M e V)^{-1} \\
\text { model } / \mathbf{e x p}\end{array}$ & $\begin{array}{c}2 \mathfrak{I}_{\text {rigflow }} / \mathrm{h}^{2} \\
(\mathrm{MeV})^{-1}\end{array}$ & $\begin{array}{c}2 \mathfrak{I}_{\text {rigbody }} / \mathrm{h}^{2} \\
(\mathrm{MeV})^{-1}\end{array}$ & $\begin{array}{c}\text { Predicted } \\
\boldsymbol{e} \boldsymbol{Q}_{o} / \boldsymbol{B}(\boldsymbol{E} 2) \\
\text { e } \mathrm{fm}^{2} / \text { w.u. }^{9}\end{array}$ & $\begin{array}{l}\text { Measured } \\
\boldsymbol{e} Q_{o} / \boldsymbol{B}(\boldsymbol{E} 2) \\
e \mathrm{fm}^{2} / \text { w.u. }\end{array}$ \\
\hline \multirow{12}{*}{${ }_{66}^{162} D y$} & $2^{+}$ & $0.08 / 0.09$ & $76 / 69$ & 89 & 2892 & $334 / 213$ & $-/ 204 \pm 3[67]$ \\
\hline & $4^{+}$ & $0.27 / 0.27$ & $76 / 78$ & 89 & 2894 & $353 / 122$ & $-/ 289 \pm 12[67]$ \\
\hline & $6^{+}$ & $0.56 / 0.55$ & $76 / 78$ & 89 & 2897 & $382 / 126$ & $-/ 301 \pm 17[67]$ \\
\hline & $8^{+}$ & $0.95 / 0.92$ & $76 / 81$ & 90 & 2901 & $422 / 146$ & $-/ 346 \pm 17[67]$ \\
\hline & $10^{+}$ & $1.45 / 1.38$ & $77 / 84$ & 90 & 2907 & $475 / 180$ & $-/ 350 \pm 23[68]$ \\
\hline & $12^{+}$ & $2.04 / 1.90$ & $77 / 88$ & 90 & 2914 & $542 / 229$ & $-/ 330 \pm 40[68]$ \\
\hline & $14^{+}$ & $2.74 / 2.49$ & 78/91 & 90 & 2922 & $625 / 301$ & $-/ 330 \pm 40[68]$ \\
\hline & $16^{+}$ & $3.53 / 3.14$ & $79 / 96$ & 91 & 2933 & $727 / 402$ & N/A \\
\hline & $18^{+}$ & $4.41 / 3.83$ & $79 / 101$ & 91 & 2946 & $843 / 1550$ & N/A \\
\hline & $20^{+}$ & $5.38 / 4.58$ & $81 / 105$ & 92 & 2963 & $1013 / 771$ & N/A \\
\hline & $22^{+}$ & $6.43 / 5.35$ & $82 / 111$ & 92 & 2985 & $1228 / 1128$ & N/A \\
\hline & $\begin{array}{c}24^{+} \\
J_{c}=14^{+}\end{array}$ & $7.54 / 6.15$ & $85 / 117$ & 93 & 3022 & $\begin{array}{c}1586 / 1875 \\
(327 /- \text { at } J=0)\end{array}$ & N/A \\
\hline \multirow{7}{*}{${ }_{68}^{168} \mathrm{Er}$} & $2^{+}$ & $0.08 / 0.08$ & $75 / 75$ & 99 & 3310 & $367 / 244$ w.u. ${ }^{10}$ & $-/ 213 \pm 4[69]$ \\
\hline & $4^{+}$ & $0.26 / 0.26$ & $76.0 / 76.0$ & 99 & 3320 & $424 / 167$ w.u. & $-/ 319 \pm 9[69]$ \\
\hline & $6^{+}$ & $0.55 / 0.55$ & $77 / 77$ & 99 & 3336 & 517/220 w.u. & $-/ 424 \pm 18[69]$ \\
\hline & $8^{+}$ & $0.94 / 0.93$ & $78 / 79$ & 100 & 3359 & 653/333 w.u. & $-/ 354 \pm 13[69]$ \\
\hline & $10^{+}$ & $1.41 / 1.40$ & $80 / 81$ & 101 & 3392 & $843 / 538$ w.u. & $-/ 308 \pm 13[69]$ \\
\hline & $12^{+}$ & $1.97 / 1.94$ & $83 / 84$ & 102 & 3438 & 1116/925 w.u. & $-/ 345 \pm 18[69]$ \\
\hline & $\begin{array}{c}14^{+} \\
J_{c}=14^{+}\end{array}$ & $2.59 / 2.57 *$ & $87 / 86$ & 105 & 3512 & $\begin{array}{c}1563 / 1791 \text { w.u. } \\
(343 / \text { - at } J=0)\end{array}$ & $-/ 336+20,-69[69]$ \\
\hline
\end{tabular}

* No measured ground-state rotational-band energy level above this energy is reported in the Table of Isotopes and Nuclear Data Sheets.

\section{References}

[1] A. Bohr, Kgl. Dan. Mat. Fys. Medd. 26 (1952) 14.

[2] A.S. Davydov and G. F. Fillippov, Nucl. Phys. 8 (1958) 237.

[3] A. Faessler and W. Greiner, Z. Physik 180 (162) 425.

[4] J.M. Eisenberg and W. Greiner, Nuclear Theory (North-Holland, Amsterdam, 1970).

\footnotetext{
${ }^{9}$ In weisskopt unit, 1 w.u. $=52.3 \mathrm{e}^{2} \mathrm{fm}^{4}$.

${ }^{10}$ In weisskopt unit, 1 w.u. $=54.9 e^{2} \mathrm{fm}^{4}$.
} 
[5] P. Ring and P. Schuck, The Nuclear Many-Body Problem (Springer-Verlag, N.Y., 1980).

[6] D.J. Rowe, Nuclear Collective Motion (Mathuen and Co. Ltd., London, 1970).

[7] H.J. Lipkin, A. de Shalit, and I. Talmi, Nuovo Cimento 2 (1955) 773.

[8] R. Skinner, Can. J. Phys. 34 (1956) 901.

[9] W. Scheid and W. Greiner, Ann. Phys. (N.Y.) 48 (1968) 493.

[10] F.M. Villars and G. Cooper, Ann. Phys. 56 (1970) 224.

[11] D.J. Rowe, Nucl. Phys. A152 (1970) 273.

[12] F.M. Villars, Ann. Phys. (N.Y.) 56 (1970) 224.

[13] B. Fink, D. Kolb, W. Scheid, and W. Greiner, Ann. Phys. 69 (1972) 375.

[14] P. Gulshani and D.J. Rowe, Can. J. Phys. 54 (1976) 970.

[15] O.L. Weaver, R.Y. Cusson, and L.C. Biedenharn, Ann. Phys. (N.Y.) 102 (1976) 493.

[16] B. Buck, L.C. Biedenharn, and R.Y. Cusson, Nucl. Phys. A317 (1979) 205.

[17] P. Gulshani, Phys. Letts. 77B (1978) 131.

[18] H. Herold, M. Reinecke, and H. Ruder, J. Phys. G: Nucl. Phys. 5 (1979) 907.

[19] P. Gulshani, J. Phys. A: Math. Gen. 14 (1981) 97.

[20] P. Gulshani and A.B. Volkov, J. Phys. A: Math. Gen. 15 (1981) 47.

[21] D. J. Rowe, Rep. Prog. Phys. 48 (1985) 1419.

[22] R.M. Lieder and H. Ryde, in Adv, Nucl. Phys.10 (1978) chapter 1, M. Baranger and E. Vogt (editors) (Plenum Press, N.Y., 1978).

[23] L. Prochniak and S.D. Rohozinski, J. Phys. G: Nucl. Part. Phys. 36 (2009) 123101.

[24] J.P. Delaroche, M. Girod, J. Libert, H. Goutte, S. Hilaire, S. Peru, N. Pillet, and G.F. Bertch, Phys. Rev. C81 (2010) 014303.

[25] M.A. Caprio, P. Maris, and J.P. Vary, Phys. Letts. B719 (2013) 179.

[26] P. Gulshani, Nuclear Physics A 832 (2010) 18.

[27] P. Gulshani, Nuclear Physics A852 (2011) 109.

[28] R.Y. Cusson, Nucl. Phys. A114 (1968) 289. 
[29] F.M. Villars and G. Cooper, Ann. Phys. 56 (1970) 224.

[30] P. Gulshani and D.J. Rowe, Can. J. Phys. 54 (1976) 970.

[31] B. Buck, L.C. Biedenharn, and R.Y. Cusson, Nucl. Phys. A317 (1979) 205.

[32] P. Gulshani and A.B. Volkov, J. Phys. A: Math. Gen. 15 (1981) 47.

[33] J.L. Birman, R.G. Nazmitdinov, and V.I. Yukalov, Phys. Rep. 526 (2013) 1.

[34] P. Gulshani, arXiv: 1407.3635v2 [nucl-th] October 24, 2014.

[35] P. Gulshani, arXiv:1401.5328 [nucl-th] January 21, 2014.

[36] S. Frauendorf, Rev. Mod. Phys. 73 (2001) 463.

[37] A. Bohr and B.R. Mottelson, Nuclear Structure, Vol. II (Benjamin, N.Y., 1975).

[38] S. M. Harris, Phys. Rev. 138B (1965) 509.

[39] M. A. J. Mariscotti, G. Scharff-Goldhaber, and B. Buck, Phys. Rev. 178 (1969) 1864.

[40] C.W. Ma and J.O. Rasmussen, Phys. Rev. C2 (1970) 798.

[41] R.A. Sorensen, Rev. Mod. Phys. 45 (1973) 353.

[42] A.V. Afanasjev, D.B. FOSSAN, G.J. LANE, and I. RAGNARSSON, Phys. Rept. 322 (1999) 1.

[43] E.R. Marshalek, Phys. Rev. 139 (1965) B770.

[44] E.R. Marshalek, Phys. Rev. 158 (1967) 993.

[45] M.A. Deleplanque, S. Frauendorf, V.V. Pashkevich, S.Y. Chu, and A. Unzhakova, Phys. Rev. C69 (2004) 044309.

[46] P. Gulshani and D.J. Rowe, Can. J. Phys. 56 (1978) 468.

[47] P. Gulshani and D.J. Rowe, Can. J. Phys. 56 (1978) 480.

[48] F.S. Stephens, in Frontiers in Nuclear Dynamics, editors: R.A. Broglia and C.H. Daso, (Plenum Press, N.Y., 1985).

[49] S.G. Nilsson and I. Ragnarsson, Shapes and Shells in Nuclear Structure (Cambridge, U.K., University Press, 1995).

[50] A. Bohr and B.R. Mottelson, Nuclear Structure, Vol. II (Benjamin, N.Y., 1975). 
[51] M. Akmali, E.S. Paul, P.J. Nolan, S.V. Rigby, M.A. Riley, and J. Simpson, in Proceedings of Conference on Nuclear Structure at the Extreme, University of the West of Scottland, Glasgow, Scottland, U.K., 2008 May 08-10.

[52] T. Tanaka, F. Sakata, T. Marumori, and K. Iwasawa, Phys. Rev. C56 (1997) 180.

[53] N.J. Stone, At. Nucl. Data Tables 90 (2003) 75.

[54] C.M. Lederer and V.S. Shirley (editors), Table of Isotopes, Seventh edition, (John Wiley and Sons, Inc., New York, U.S.A., 1978).

[55] D. Brandford, A.C. McGouph, and I.F. Weight, Nucl. Phys. A241 (1975) 349.

[56] Y. Abgrall, G. Baron, E. Caurier, and G. Monsonego, Nucl. Phys. A131 (1969) 609.

[57] Y. Abgrall, B. Morand, and E. Caurier, Nucl. Phys. A192 (1972) 372.

[58] S. Das Gupta and M. Harvey, Nucl. Phys. A94 (1967) 602.

[59] J. Zofka and G. Ripka, Nucl. Phys. A168 (1971) 65.

[60] M. Bouten and M.C. Bouten, Nucl. Phys. A193 (1972) 49.

[61] K.R. Lassey, M.R.P. Manning, and A.B. Volkov, Can. J. Phys. 51 (1973) 2522.

[62] J. Pelet and J. Letourneux, Nucl. Phys. A281 (1977) 277.

[63] M.G. Vassanji and M. Harvey, Nucl. Phys. A344 (1980) 61.

[64] J.P. Draayer, K.J. Weeks, and G. Rosensteel, Nucl. Phys. A413 (1984) 215.

[65] G. Rosensteel, J.P. Draayer, and K.J. Weeks, Nucl. Phys. A419 (1984) 1.

[66] S. Raman, C.W. Nestor, Jr., and P. Tikkanen, At. Nucl. Data Tables 78 (2001) 1.

[67] Y. Horikawa, Progr. Theor. Phys. 47 (1972) 867.

[68] C.W. Reich, Nucl. Data Sheets 108 (2007) 1807.

[69] C.M. Baglin, Nucl. Data Sheets 111 (2010) 1807.

[70] A. Faessler, W. Greiner, and R.K. Sheline, Nucl. Phys. 62 (1965) 241.

[71] S.G. Nilsson and O. Prior, Mat. Fys. Medd. Dan. Vid. Selsk. 32 (1961) No. 16. 